\title{
Artrite reumatóide e doença cardiovascular na atualidade: o que sabemos sobre essa associação e o que podemos fazer pelo paciente?
}

\author{
Rheumatoid arthritis and cardiovascular disease: \\ what do we know about this relationship and how \\ can we treat affected patients nowadays?
}

\author{
Michel Haddad', Aline Domingos Pinto Ruppert', \\ Alexandre de Matos Soeiro ${ }^{2}$, Carlos V. Serrano $\mathrm{Jr}^{3}$
}

\begin{abstract}
Haddad M, Ruppert ADP, Soeiro AM, Serrano Jr CV. Artrite reumatóide e doença cardiovascular na atualidade: O que sabemos sobre essa associação e o que podemos fazer pelo paciente?/ Rheumatoid arthritis and cardiovascular disease: What do we know about this relationship and how can we treat affected patients nowadays? Rev Med (São Paulo). 2012 abr.-jun.;91(2):87-95.
\end{abstract}

RESUMO: O interesse em doenças auto-imunes vem crescendo a cada ano, principalmente sobre sua relação com doenças cardiovasculares. Especificamente a artrite reumatóide vem sendo considerada um fator de risco independente para doença arterial coronária nos últimos anos. Diversos estudos foram realizados recentemente com o objetivo de esclarecer pontos cruciais na estratificação de risco desses pacientes e no seu respectivo tratamento medicamentoso adequado. Novas terapias específicas da doença reumatóide ainda estão em estudo, e prometem reduzir o risco cardiovascular a longo prazo. Desse modo, realizamos uma revisão bibliográfica ampla, utilizando as principais bases de dados nacionais e internacionais, com o objetivo de salientar a importância de mecanismos ateroscleróticos e inflamatórios sobre a doença arterial coronária. Além disso, frente às atuais evidências, sugerimos estratégias de estratificação de risco e tratamento da doença arterial coronária em pacientes com artrite reumatóide.

DESCRITORES: Artrite reumatóide/fisiopatologia; Artrite reumatóide/terapia; Doenças cardiovasculares

\begin{abstract}
The interest in auto-immune diseases has been grown, mainly about cardiovascular illnesses. Rheumatoid arthritis has been considered an independent risk factor for coronary arterial disease in last years. Recently, different studies have been performed in order to better clarify crucial points in risk stratification and correct treatment for patients with rheumatoid arthritis. New specific therapies have been studying and promising reduction of cardiovascular risk. Thereby, we made a bibliographic revision to show the importance of atherosclerotic and inflammatory mechanisms in coronary artery disease. Furthermore, we suggested different strategies to establish risk stratification and treatment of cardiovascular diseases in patients with rheumatoid arthritis.
\end{abstract}

KEYWORDS: Arthritis, rheumatoid/physiopathology; Arthritis, rheumatoid/therapy; Cardiovascular diseases

Prêmio Monografias - COMU 2011.

1. Acadêmicos do Curso de Medicina da Faculdade de Medicina da Universidade de São Paulo. 2. Residência em Clínica Médica e Cardiologia, Instituto do Coração do Hospital das Clínicas da Faculdade de Medicina da Universidade de São Paulo. 3.Médico Instituto do Coração do Hospital das Clínicas da Faculdade de Medicina da Universidade de São Paulo. Endereço para correspondência: Aline Domingos Pinto Ruppert. Rua Oscar Freire, 2121-Pinheiros, São Paulo, SP.E-mail: alinerup@ gmail.com 


\section{INTRODUÇÃO}

artrite reumatóide (AR) é uma doença inflamatória crônica que acomete de 0,2\% a 2,0\% da população brasileira ${ }^{1-3}$, preferencialmente mulheres, com pico de incidência entre os 30 e 50 $a{ }^{4}$. Nos últimos anos, diferentes estudos têm demonstrado que pacientes com AR apresentam de 5 a 10 anos a menos de expectativa de vida em relação à população em geral. Essa diminuição está relacionada ao maior risco de doenças cardiovasculares, que é de 2 a 5 vezes maior que na população em geral2,3,5,6. Dessa forma, o risco cardiovascular absoluto em portadores de AR é equivalente ao dos indivíduos sem AR que são 5 a 10 anos mais velhos ${ }^{6-8}$. Embora o aumento do risco relativo (RR) de eventos cardiovasculares seja mais pronunciado em pacientes mais jovens, são os pacientes mais velhos portadores de AR que sofrem, em termos absolutos, um número maior de eventos, principalmente devido ao desenvolvimento de doença arterial coronária (DAC) precoce ${ }^{2}$. Coorte prospectiva realizada no EUA em 609 pacientes com AR durante um seguimento médio de 14 anos demonstrou risco maior de morte em indivíduos com AR em comparação à pacientes sem AR da mesma idade e sexo (Figura 1), com maior propensão à ocorrência de eventos cardiovasculares $(\mathrm{RR}=1,6$; $\mathrm{p}<0,001)^{8}$.

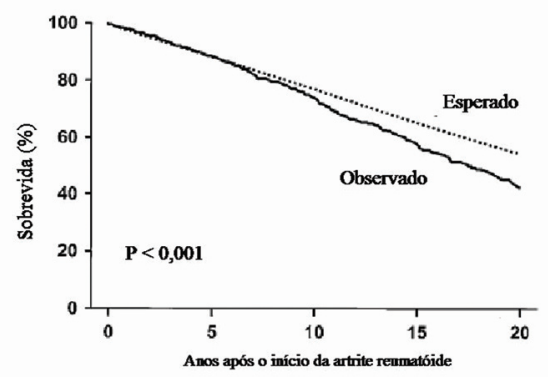

Figura 1. Sobrevida de indivíduos com artrite reumatóide em comparação à sobrevida esperada da população em geral (Adaptado de Gabriel et al. Arthritis Rheum 2003;48:54-58) ${ }^{8}$

Desse modo, nessa revisão faremos um resumo das últimas publicações científicas a respeito da fisiopatologia da aterosclerose em pacientes com $A R$, incluindo considerações sobre a contribuição dos fatores de risco clássicos e elucidações sobre mecanismos inflamatórios, específicos da AR. Além disso, serão analisados os últimos estudos clínicos acerca da ocorrência de eventos cardiovasculares em $\mathrm{AR}$, discutindo estratégias de estratificação de risco e tratamento adequado da DAC na AR.

\section{Fisiopatologia e aterosclerose na AR}

Há crescente evidência, por estudos clínicos controlados, de que pacientes com AR apresentam aterosclerose e calcificação coronária mais extensa em relação a indivíduos controles sem AR, sugerindo que este aumento de eventos cardiovasculares seria reflexo de maior e mais precoce atividade aterosclerótica ${ }^{9}$. Foi demonstrado, pela avaliação do escore de cálcio, que estes pacientes apresentam precocemente maior calcificação em artéria aorta, artérias carótidas e artérias coronárias quando comparados com o grupo controle 6 . Além disso, foi também observado que a maior calcificação arterial nesses pacientes é mais pronunciada em indivíduos do sexo masculino e em faixas etárias inferiores, e que a maior extensão de calcificação coronária está associada a casos mais severos de artrite reumatóide ${ }^{10}$.

A AR é considerada fator de risco independente para aumento da espessura das camadas íntima e média das artérias carótidas comuns e femorais, fato que se correlacionou com gravidade e cronicidade da doença reumatológica ${ }^{3,11}$. O motivo pelo qual ocorre aterosclerose precoce e mais intensa em pacientes com AR tem sido alvo de intensas pesquisas. Recentemente vem sendo aceito que a inflamação crônica, presente na $A R$, representa um papel importante na gênese e desenvolvimento das placas ateroscleróti$\operatorname{cas}^{10}$. O estado de inflamação vascular crônico, com o aumento de citocinas circulantes e a presença de auto-anticorpos, pode promover instabilidade da placa aterosclerótica aumentando seu risco de ruptura. O mesmo substrato de linfócitos T CD4 e CD8 encontrados em placas instáveis também é observado em lesões ósseas erosivas de AR e em pacientes com vasculites. Além disso, granzima $B$, um produto de macrófagos envolvido no remodelamento da matriz extracelular, é observada em grande quantidade tanto na sinóvia de pacientes com AR quanto em áreas de ruptura de placas ateroscleróticas, reforçando a idéia de inflamação sistêmica como causa de maior risco de eventos cardiovasculares ${ }^{2}$.

Outras moléculas sabidamente presentes na fisiopatologia da AR também estão sendo relacionadas à doença aterosclerótica. É o caso das metaloproteinases da matriz, enzimas proteolíticas que não só participam da destruição articular como também atuam na desestabilização e rotura de placas ateroscleróticas vulneráveis. De forma similar, níveis aumentados de fatores de coagulação e citocinas inflamatórias como fibrinogênio, fator tecidual, fator de von Willebrand, inibidor de ativação do plasminogênio, proteína-C reativa (PCR), interferon-gama, fator de necrose tumoral- $\alpha$ (TNF- $\alpha$ ), interleucina-6 (IL-6) e interleucina-I beta são encontrados tanto na AR quanto na doença coronariana ${ }^{5,11-13}$. Além disso, outra molécula que encontra-se em níveis elevados em pacientes com AR é o óxido nítrico, produzido 
Haddad M, et al. Artrite reumatóide e doença cardiovascular na atualidade.

por células endoteliais e responsável em parte por mecanismos de apoptose e regulação de linfócitos T em placas ateroscleróticas ${ }^{12}$.

Ponto crucial para o desenvolvimento de placas ateroscleróticas refere-se à função endotelial ${ }^{12}$. O endotélio tem um importante papel na regulação do tônus vascular, atividade plaquetária e trombogênese. A disfunção endotelial é um dos primeiros passos para o surgimento de placas ateroscleróticas. Estados inflamatórios sistêmicos como AR podem levar à disfunção endotelial, principalmente devido aumento de citocinas circulantes. Alguns estudos mostraram a presença indireta de disfunção endotelial, uma vez que pacientes com AR apresentam menor vasodilatação dependente de endotélio em relação à indivíduos sem $\mathrm{AR}^{12}$.

\section{Importância dos fatores de risco clássicos em pacientes com AR}

Além dos discutidos efeitos aterogênicos da inflamação sistêmica crônica, os fatores de risco clássicos, como tabagismo, hipertensão arterial sistêmica (HAS), diabetes mellitus (DM) e dislipidemia também atuam na gênese da doença aterosclerótica em pacientes portadores de $\mathrm{AR}^{14}$. Diversos estudos examinaram a prevalência dos fatores de risco clássicos em pacientes portadores de AR, e sua participação na gênese de doenças cardiovasculares. Em comparação aos grupos controle, foi evidenciado que nestes pacientes não há diferenças significativas na prevalência de HAS, DM, dislipidemia e obesidade, no período de inicio da artrite reumatóide. Dos fatores de risco clássicos, apenas o tabagismo se mostrou mais prevalente entre indivíduos portadores de AR estando relacionado a maior risco de doença cardiovascular antes mesmo do surgimento da doença inflamatória6,11,12,15.

Esses indivíduos apresentam, ao longo do curso da AR, menor risco de desenvolver hiperlipidemia, e menores índices de massa corpórea (IMC) que a população em geral ${ }^{6,7}$. A prevalência e a caracterização da dislipidemia nesses pacientes permanecem pouco conhecidas, com diversos estudos mostrando resultados controversos. Alguns autores encontraram níveis séricos diminuídos de HDL-colesterol e aumentados de LDL-colesterol nesses pacientes ${ }^{11,16}$. Paradoxalmente, outros autores caracterizaram um perfil lipídico com colesterol total, LDL-colesterol, HDL-colesterol diminuídos e aumento de triglicérides, correlacionados diretamente com a atividade inflamatória sistêmica ${ }^{2,11}$. Reforçando essa última corrente, há evidência de que o perfil lipídico tenha um efeito paradoxal no risco cardiovascular em pacientes com $A R$, já que se mostrou que os níveis de colesterol total e LDL-colesterol diminuem bruscamente no período de 3 a 5 anos que precede a incidência da $A R^{6,17}$. Portanto, valores menores de colesterol total e LDL-colesterol corresponderiam a um maior risco cardiovascular nesses pacientes devido à suposta maior atividade inflamatória associada. Além disso, alguns estudos observaram maiores níveis de lipoproteína (a) sérica, que em parte poderia também estar estar associada a um maior risco, porém ainda sem comprovação ${ }^{11,12}$.

Diferentemente da população em geral, um baixo IMC $\left(<20 \mathrm{~kg} / \mathrm{m}^{2}\right)$ em indivíduos com AR está associado a um risco importante de morte cardiovascular. Como o baixo IMC nestes indivíduos pode indicar maior atividade inflamatória sistêmica, este achado corrobora a hipótese da importância da inflamação no risco cardiovascular nestes pacientes ${ }^{6,7}$. Além disso, limitações funcionais devido deformidades ósseas próprias da doença fazem com que haja uma maior tendência à pessoas com AR serem sedentárias, o que também contribuiria para aumento do risco cardiovascular nesses pacientes ${ }^{12}$.

Um importante fator de risco adicional encontrado em pacientes com AR é o aumento da resistência à insulina e síndrome metabólica, algo diretamente relacionado ao aumento da espessura da camada íntima-média da carótida e níveis elevados de escore de cálcio coronariano nesses pacientes ${ }^{2}$. $\mathrm{O}$ aumento da resistência à insulina seria devido ao estado inflamatório crônico prejudicando a função das células beta pancreáticas ${ }^{11}$. O TNF- $\alpha$ também seria um importante mediador nesse processo, por inibir a captação de glicose no músculo esquelético ${ }^{11}$.

Curiosamente, apesar de apresentarem prevalência semelhante à população em geral, o impacto relativo desses fatores de risco clássicos se mostrou ser pronunciadamente menor em pacientes com AR, ou seja, o risco da ocorrência de eventos cardiovasculares relacionada a esses fatores é muito menos relevante nestes pacientes. Provavelmente esse fato deve-se à presença de um forte componente de inflamação sistêmica como fator de risco para doenças cardiovasculares nos pacientes com AR, o que diluiria o efeito dos outros fatores de risco ${ }^{2,6,7}$. O escore de risco de Framingham amplamente utilizado na prática clínica foi validado em pacientes com AR e pode ser utilizado nesses pacientes, de preferência sendo complementado com algum método auxiliar de imagem não invasivo para estratificação de risco, uma vez que escore de risco de Framingham elevado mostrou ser um forte preditor 
de calcificação coronária em pacientes com $A R^{9,16}$.

\section{Marcadores inflamatórios associados a risco cardiovascular}

Como já comentado anteriormente, é fato a existência de fatores inflamatórios crônicos que contribuam para o desenvolvimento da placa aterosclerótica em pacientes com AR (Tabela 1). Dentre esses, nos últimos anos têm sido feitas tentativas de isolar marcadores inflamatórios mensuráveis, capazes de predizer risco cardiovascular nessa população ${ }^{6}$. Dessa forma, altos níveis de velocidade de hemossedimentação (VHS) (> $40 \mathrm{~mm} / \mathrm{h})$, acometimento de pequenas e médias articulações, erosões ósseas, nódulos reumatóides, vasculites e doença reumatóide pulmonar já foram associadas com maior risco de morte $2,6-8,18,19$.

Tabela 1. Mediadores inflamatórios associados à aterosclerose na artrite reumatóide

\begin{tabular}{ll}
\hline $\begin{array}{l}\text { Mediador } \\
\text { inflamatório }\end{array}$ & $\begin{array}{l}\text { Contribuição na gênese da doença } \\
\text { aterosclerótica em pacientes com artrite } \\
\text { reumatóide }\end{array}$ \\
\hline
\end{tabular}

\begin{tabular}{|c|c|}
\hline IL-1, TNF- $\alpha$ & $\begin{array}{l}\text { Iniciam o processo de lesão das células en- } \\
\text { doteliais, induzindo a expressão de moléculas } \\
\text { de adesão nestas células }\end{array}$ \\
\hline PCR & $\begin{array}{l}\text { Tem o mesmo efeito de IL-1 e TNF- } \alpha \text {, e valores } \\
\text { maiores desta molécula estão associados à } \\
\text { maior calcificação coronariana em mulheres } \\
\text { com artrite reumatóide }{ }^{5}\end{array}$ \\
\hline IL-6 & $\begin{array}{l}\text { Maiores valores dessa molécula também estão } \\
\text { associados à maior calcificação coronariana } \\
\text { em pacientes com artrite reumatóide, porém } \\
\text { a associação é parcial, não sendo tão forte } \\
\text { quanto a da } \text { PCR }^{21}\end{array}$ \\
\hline ADMA & $\begin{array}{l}\text { Inibe a NO sintase, reduzindo a concentração } \\
\text { de óxido nítrico e contribuindo para a lesão } \\
\text { endotelial }{ }^{10}\end{array}$ \\
\hline MMP's & $\begin{array}{l}\text { Desestabilizam e rompem placas ateroscle- } \\
\text { róticas vulneráveis }{ }^{5}\end{array}$ \\
\hline
\end{tabular}

Legenda: * IL-1 = interleucina-1; TNF- $\alpha=$ fator de necrose tumoral- $\alpha$; PCR = proteína-C reativa; IL-6 = interleucina-6; ADMA = dimetil-arginina; MMP's = metaloproteinases.

Marcador extensamente pesquisado é a presença de fator reumatóide (FR). Em diferentes estudos, níveis elevados de FR estiveram diretamente relacionados à maior risco de morte e de eventos combinados como infarto agudo do miocárdio (IAM), insuficiência cardíaca e doença vascular periférica ${ }^{6,20}$. Estudo realizado por Gabriel et al. ${ }^{7}$ mostrou RR de morte cardiovascular em mulheres com AR e FR negativo de 1,59 (IC 95\%, 1,14-2,15) comparado ao risco em mulheres com FR positivo de 2,10 (IC 95\%, 1,73-2,51). Em homens, obteve resultado semelhante com RR de 1,59 (IC 95\%, 1,19-2,09) quando FR positivo e 0,81 (IC 95\%, 0,41-1,46) quando negativo ${ }^{7}$. Alguns autores sugerem ainda que mesmo na ausência de AR, pessoas com FR positivo possuem maior risco de eventos cardiovasculare ${ }^{20}$.

Considerações semelhantes foram encontrados em pacientes com AR e fator anti-núcleo (FAN) positivo, estando também relacionado a maior risco de eventos cardiovasculares ${ }^{7,20}$.

Em relação à $P C R$, também demonstrouse que níveis elevados aumentam o risco de eventos cardiovasculares em pacientes com AR. Sua contribuição é essencial na fisiopatologia da aterosclerose, já que o ajuste por níveis séricos dessa molécula anula a relação entre calcificação coronariana e AR em mulheres $2,5,6,11$.

Além do VHS, FR, FANePCR, outrosmarcadores que se relacionam com evento cardiovascular incluem IL-6, moléculas intercelulares de adesão do tipo I solúveis, amilóide $A$ e fibrinogênio ${ }^{11,12}$. A IL-6 também atua como importante mediador, uma vez que o ajuste por níveis séricos dessa interleucina atenua a relação entre calcificação coronariana e AR, indicando uma participação parcial dessa molécula ${ }^{21}$.

\section{Características da doença arterial coronária em pacientes com AR}

Pacientes com AR têm risco elevado de síndromes coronarianas agudas. Segundo observação do estudo Rochester ${ }^{15}$ que acompanhou 603 pacientes com AR, especificamente no período de 2 anos que precedem o aparecimento da AR esses pacientes estão mais propensos à ocorrência de IAM (Odds Ratio [OR] = 3,17, IC 95\%, 1,16-8,68) e isquemia "silenciosa" (OR = 5,86, IC 95\%, 1,29-26,64) em comparação à indivíduos da população em geral do mesmo sexo e idade sem $A R^{2,3,7,15,16,22}$. Após 0 diagnóstico, o risco elevado de isquemia "silenciosa" persiste (OR =2,13, IC 95\%, 1,13-4,03), assim como a maior ocorrência de morte súbita (OR $=1,94$, IC $95 \%, 1,05-3,55)$, como observado na Figura $2^{3,7,15,22}$. Quanto maior o tempo de duração da doença, maior o risco de ocorrência de eventos coronarianos, principalmente após 10 anos do diagnóstico ${ }^{16}$.

Além disso, foi evidenciado em diversos estudos que o padrão de doença cardiovascular nos pacientes com AR é diferente do observado na população em geral. Estes pacientes apresentam menos sintomas de DAC, como angina pectoris, estando, portanto, mais propensos a desenvolverem isquemia "silenciosa" e morte súbita7. Esta particularidade pode estar associada à maior produção de citocinas pró 
Haddad M, et al. Artrite reumatóide e doença cardiovascular na atualidade.

e anti-inflamatórias por indivíduos com AR, o que alteraria a percepção da dor ${ }^{7,15}$. Pode, também, ter relação à menor mobilidade destes pacientes, devido à doença articular, o que os impediria de realizar esforço que desencadeasse angina, ou à menor valorização da dor por parte tanto do médico quanto do próprio paciente, que atribuem sua origem como osteomuscular relacionada à $A R^{7,11}$.

Do ponto de vista anatômico, estudo realizado
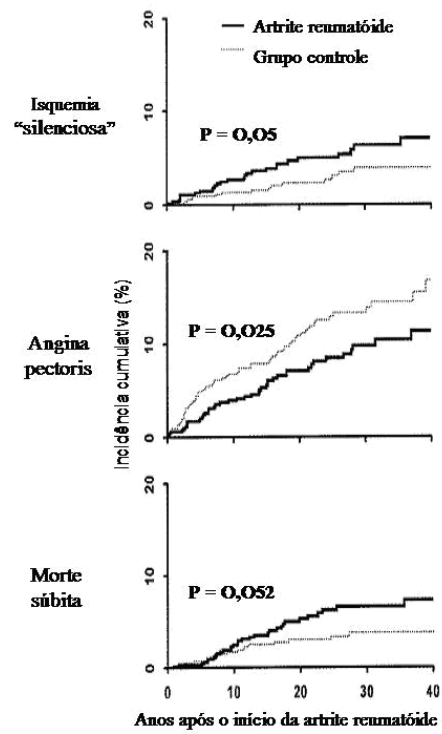

Figura 2. Incidência cumulativa de isquemia "silenciosa", angina e morte súbita em pacientes com artrite reumatóide, comparados à indivíduos controles (Adaptado de Maradit-Kremers et al. Arthritis Rheum 2005;52:402-11 ${ }^{15}$

em autópsias de pacientes com AR não encontrou diferenças quanto à extensão e ao grau de estenose da lesão coronariana quando comparada à indivíduos sem AR. Ao contrário do esperado, poucos pacientes com AR apresentavam doença triarterial em comparação à indivíduos sem AR (32\% x 61\%; p $<0,018$ ) e a doença aterosclerótica tendia a ser até mesmo menor $^{23}$. No entanto, observou maior atividade inflamatória em placas ateroscleróticas presentes nas artérias circunflexa e descendente anterior, com $48 \%$ das placas em descendente anterior sendo classificadas como vulneráveis, em comparação à apenas 22\% em pacientes sem AR $(p=0,018)^{7,23}$. Novamente, esse estudo sugere mais uma vez que a etiologia da DAC em pacientes com AR esteja mais relacionada à inflamação ${ }^{7,23}$.

\section{Doença extra-coronariana em pacientes com AR}

Diferentes estudos têm demonstrado que pacientes com AR possuem maior espessamento da camada íntima-média carotídea. Além disso, a progressão da placa aterosclerótica tende a ser maior em pacientes com AR e diretamente relacionada aos níveis plasmáticos de PCR e VHS. O risco de ocorrência de placas ateroscleróticas em carótidas de pacientes com AR chega a ser até três vezes maior do que em indivíduos sem AR, mesmo quando ajustado para idade, sexo e demais fatores de risco cardiovasculares ${ }^{16,24}$.

De maneira distinta do observado em relação à $\mathrm{DAC}$, em nenhum dos grandes estudos de coorte realizados em pacientes com AR foi observada maior incidência de acidente vascular cerebral ou doença arterial periférica ${ }^{7,16}$.

\section{AR e sua relação com o desenvolvimento de in-} suficiência cardíaca

Assim como na DAC, pacientes com AR possuem risco elevado de desenvolvimento de insuficiência cardíaca em comparação à população em geral ${ }^{7,19,25}$. A incidência cumulativa de insuficiência cardíaca em 30 anos de seguimento chega a $34 \%$ em pacientes com AR em comparação à $25 \%$ no restante da população $(p<0,001)$, mesmo após ajuste para diferentes fatores de risco cardiovasculares ${ }^{7,13,15,19,25}$. No entanto, o risco de insuficiência cardíaca parece estar diretamente relacionado à presença de fator reumatóide positivo (OR = 2,59, IC 95\%, 1,95-3,43) quando comparado a pacientes com fator reumatóide negativo $(O R=2,57 \text {, IC 95\%, 1,20-5,49) })^{7,15,19}$.

Estudo de coorte prospectivo com seguimento médio de 15 anos realizado por Maradit-Kremers et al. ${ }^{18}$ em 575 pacientes com AR mostrou que nos 6 meses que precedem o aparecimento da insuficiência cardíaca, cerca de $23 \%$ dos pacientes apresentavam VHS $>40 \mathrm{~mm} / \mathrm{h}$, número esse elevado comparado à média de apenas $10 \%$ encontrada durante todo 0 seguimento do restante dos pacientes. Segundo os autores, isso sugere que da mesma forma que na doença coronariana um estímulo inflamatório possa estar envolvido no início da insuficiência cardíaca em pacientes com $\mathrm{AR}^{18}$.

Além disso, observou-se que o prognóstico da insuficiência cardíaca em pacientes com AR é pior do que em indivíduos sem a doença. No período de 30 dias após o diagnóstico de insuficiência cardíaca, a mortalidade nos pacientes com AR chega à $16 \%$, comparado à $7 \%$ nos indivíduos sem $\operatorname{AR}(p<0,001)$. A mesma tendência de mortalidade também observase após 6 meses de seguimento no grupo AR (OR = 1,94, IC 95\%, 1,17-3,23)

Por último, pacientes com AR apresentam maior chance de desenvolverem insuficiência cardíaca com fração de ejeção de ventrículo esquerdo 
preservada comparado à indivíduos sem AR (OR $=2,57, \mathrm{IC} 95 \%, 1,20-5,49)^{7,26}$. Estudo recente com seguimento médio de 25 anos mostrou que após esse período cerca de $31 \%$ dos pacientes com AR apresentavam diagnóstico de insuficiência cardíaca com fração de ejeção preservada (> 50\%) em comparação à $26 \%$ dos indivíduos sem AR da mesma idade e sexo (OR = 1,6, IC 95\%, 1,2-2,4). Além disso, indivíduos com AR possuíam de maneira significativa menor índice de massa de ventrículo esquerdo, maior valor de pressão arterial pulmonar e maior volume de átrio esquerdo. O tempo de duração da AR e os níveis plasmáticos de IL-6 foram fatores independentes diretamente relacionados à maior ocorrência de insuficiência cardíaca diastólica em pacientes com $\mathrm{AR}^{26}$.

\section{Utilização de métodos diagnósticos para estrati- ficação de risco em AR}

A utilização de métodos diagnósticos de imagem auxiliares na estratificação de risco coronariano em pacientes com AR vem sendo amplamente estudada $9,14,21,24,27$. Especificamente a angiotomografia computadorizada de artérias coronárias mostrou ser útil para estratificação de risco em diferentes estudos. De maneira geral, os pacientes com AR apresentam maior calcificação de artérias coronárias, e conseqüentemente maior escore de cálcio, principalmente dependendo diretamente do tempo de duração da doença e dos níveis plasmático de $\mathrm{VHS}^{14,16}$. Estudo realizado por Wang et al. ${ }^{24} \mathrm{em} 85$ pacientes com AR submetidos à angiotomografia coronariana mostrou que esse grupo de pacientes apresenta alto RR de calcificação da aorta (OR = 19,5, IC 95\%, 8,0-47,6), carótidas (OR $=5,7, \mathrm{IC} 95 \%, 1,7-18,7)$ e artérias coronárias (OR = 5,0, IC 95\%, 2,2-11,1), em comparação à indivíduos sem $A R$ de mesma idade e sexo. Entre os pacientes com AR maiores de 60 anos, 90\% possuíam calcificação arterial difusa principalmente em aorta torácica ${ }^{24}$.

Outros estudos mostraram que a prevalência de calcificação coronária observada através da angiotomografia coronária em AR é maior em homens o que em mulheres $(p=0,012)$. Além disso, a média do escore de cálcio em pacientes com AR tende a ser 53 unidades maior do que em indivíduos sem AR que também possuem escore aumentado $(p=0,017)^{21}$.

A ultrassonografia de carótidas com medida da camada íntima-média também tem se mostrado um marcador precoce de aterosclerose com alta correlação com presença de DAC em pacientes com $A R^{10,16}$. De maneira semelhante à angiotomografia, $a$ ultrassonografia tem mostrado maior espessamento da camada íntima-média em pacientes com AR, e esse índice tende a ser maior, quanto maior a duração da doença ${ }^{10}$.

\section{Qual o efeito dos medicamentos específicos para AR sobre o risco cardiovascular?}

Existe uma dificuldade muito grande em se analisar o efeito de diferentes classes de medicamentos utilizados para o tratamento da AR sobre o risco cardiovascular, principalmente devido a fatores de confusão como indicações e contraindicações dos respectivos tratamentos. Portanto, a contribuição da maioria das medicações ainda é controversa? ${ }^{7}$.

O uso de prolongado de corticóides é implicado como sendo um potencial causador de aterosclerose precoce, resistência à insulina, HAS, aumento de colesterol total, LDL-colesterol e triglicérides, com conseqüente elevação do risco de doença cardiovascular em pacientes com $\mathrm{AR}^{2,7,11}$. No entanto, alguns estudos mostraram que, em pacientes que utilizaram doses cumulativas elevadas de corticóides (> 7000 $\mathrm{mg}$ ), o que realmente esteve associado a maior risco de eventos foi a presença de FR positivo, sendo que o uso de corticóide em si não se mostrou como fator de risco independente ${ }^{7,28}$. Além disso, em pacientes com antecedente de DAC e AR, o tratamento com corticóide parece até mesmo atenuar o risco cardíovascular, mostrando que talvez o mais importante seja o completo controle do fator inflamatório ${ }^{2,7,11}$. Parece que o uso de corticóide é capaz de reduzir os níveis de homocisteína, podendo ser outro mecanismo responsável por redução de risco cardiovascu$\mid a r^{11}$. Porém, outro estudo mostrou aumento do risco de formação de placa aterosclerótica em carótidas e doença arterial periférica em pacientes com AR que utilizaram mais de $16000 \mathrm{mg}$ de corticóide, atentando cuidados com a dose da medicação em excesso ${ }^{16}$.

O metotrexate é outra medicação que parece alterar o risco cardiovascular em pacientes com AR, embora a natureza desse seu efeito ainda seja incerta ${ }^{7,29-31}$. No estudo mais citado na literatura, o uso de metotrexate parece estar associado à redução de risco, principalmente devido grande diminuição dos casos de IAM nos pacientes que utilizaram a medicação $(\mathrm{OR}=0,3 \text {, IC 95\%, 0,2-0,7) })^{7,31-33}$. De maneira semelhante, outros estudos têm mostrado redução de doença vascular periférica em pacientes com AR que utilizaram metotrexate ${ }^{7,11,16}$. Os níveis de HDLcolesterol e apolipoproteína A parecem alterar-se favoravelmente após 12 meses de uso da medicação ${ }^{29}$. No entanto, em outro estudo em pacientes com AR e DAC estabelecida, o uso de metotrexate esteve associado a maior mortalidade $(\mathrm{OR}=3,4, \mathrm{p}$ $=0,0054)$, mostrando que ainda é necessário maior número de pesquisas nesse campo ${ }^{7}$. De maneira 
Haddad M, et al. Artrite reumatóide e doença cardiovascular na atualidade.

ainda incerta, sabe-se que o uso de metotrexate pode levar à hiperhomocisteinemia, o que supostamente aumentaria o risco cardiovascular. Além disso, o metotrexate apresenta toxicidade direta sobre as células endoteliais podendo levar à oxidação do LDLcolesterol e tendo efeitos trombóticos ${ }^{11}$.

Novas evidências têm surgido a respeito do uso de agentes anti-TNF- $\alpha$, incluindo melhor controle da inflamação sistêmica e efeitos endoteliais diretos $^{2,10,12,13,34}$. Apesar disso, uma parte dos dados ainda são conflitantes. Alguns estudos têm sugerido melhora da função endotelial com a melhora da inflamação sistêmica ${ }^{2,10,34}$. Outros referem aumento da reserva de fluxo coronário ao final de 24 meses de tratamento ${ }^{10}$. Apesar de um estudo ter mostrado haver piora do perfil lipídico a longo prazo, com redução do HDL-colesterol e aumento do colesterol total, outro estudo com seguimento de 80 pacientes em uso da medicação por 48 meses não mostrou nenhuma alteração significativa nos níveis lipídicos ao final do período 2,35 .

O uso de estatinas devido seu efeito pleiotrópico como potencial "protetor" do endotélio ainda é incerto. É amplamente aceito que pacientes com AR e LDL-colesterol elevado têm indicação de uso de estatinas. No entanto, em pacientes em estado inflamatório crônico não se sabe se os mesmos benefícios encontrados na população em geral devido à redução do LDL-colesterol podem ser esperados para esses pacientes ${ }^{2}$. A ezetimiba e sinvastatina foram testadas em pacientes com AR, nos quais observou-se redução na inflamação sistêmica, com concomitante melhora da função endotelial ${ }^{10,36}$.

A hidroxicloroquina parece reduzir o risco de desenvolvimento de DM a longo prazo em pacientes com $\mathrm{AR}^{2}$. A sulfassalazina, leflunomida e cloroquina foram testadas em um grande estudo com pacientes com AR e também mostraram redução significativa nos casos de IAM nos pacientes que receberam a medicação ${ }^{16,33}$.

\section{O que o médico cardiologista deve saber frente a um paciente com AR?}

Conforme comentado anteriormente, alguns aspectos presentes em pacientes com AR são diferentes do que seria esperado em indivíduos na população em geral. Portanto, é comum que durante a avaliação cardiológica de um paciente com AR, o médico cardiologista apresente dúvidas freqüentes. Não existem regras bem estabelecidas do que deve ser realizado nesses pacientes. De maneira simplificada, frente às atuais evidências, algumas sugestões podem ser adotadas:

1) A AR deve ser encarada como um fator de risco cardiovascular independente. É prudente que o médico lembre-se que cada paciente avaliado deve ser considerado como se tivesse 5 a 10 anos além de sua verdadeira idade;

2) Fatores de risco clássicos para aterosclerose como tabagismo, dislipidemia, HAS, DM e obesidade devem ser controlados e tratados da mesma maneira de que em qualquer outro paciente sem AR. A importância relativa desses fatores de risco em pacientes com AR é a mesma que no restante da população, acrescentando-se o fato reconhecido do desenvolvimento de aterosclerose precoce ${ }^{11}$;

3) $\mathrm{Na}$ estratificação de risco cardiovascular o escore de Framinghan foi devidamente validado em pacientes com AR, e pode ser utilizado. Nesses pacientes ele mostrou ter alto valor preditivo positivo para calcificação coronariana, quando o paciente foi classificado como de alto risco';

4) Complementando a estratificação de risco, métodos não invasivos como angiotomografia computadorizada de coronárias e ultrassonografia de carótidas foram validados nessa população e podem ser utilizados. A principio a recomendação é a mesma da população em geral ${ }^{37}$. No entanto, conforme descrito a prevalência de doença aterosclerótica subclínica em pacientes com AR é maior e, portanto, sugere-se que tais métodos sejam realizados de maneira mais precoce;

5) Não existe nenhuma recomendação específica quanto ao uso de estatinas e/ou AAS em prevenção primária para pacientes com $A R$, devendo ser adotada a mesma recomendação aplicada no restante dos pacientes ${ }^{37}$;

6) O médico cardiologista deve solicitar rotineiramente e estar atento à sintomas e/ou exames que mostram a presença de atividade inflamatória sistêmica, tais como artrite, PCR e VHS. Para todos os pacientes, o acompanhamento conjunto com o médico reumatologista é essencial, devendo-se rapidamente encaminhar o paciente com sinais de atividade inflamatória presentes $s^{6,7,20}$;

7) Fatores reconhecidos como preditores de alto risco de eventos coronarianos como FR e FAN devem ser solicitados, sendo dada atenção especial aos pacientes com esses marcadores presentes, inclusive com solicitação de métodos complementares não invasivos na estratificação de risco $0^{6,7,20}$;

8) Corticóides devem ser utilizados desde que haja real indicação de uso devido a presença de atividade inflamatória sistêmica. No entanto, a dose usada deve ser a menor possível, principalmente a longo prazo 7,28 ;

9) Medicamentos específicos como cloroquina, leflunomida, metotrexate e anti-TNF podem ser utilizados sem restrições. As atuais evidências 
mostram que em relação a desfechos clínicos o completo controle da atividade inflamatória parece ser mais importante que quaisquer efeitos colaterais das medicações ${ }^{7}$;

10) Pacientes com AR devem ser constantemente reavaliados quanto à sintomas de insuficiência cardíaca, e tratados adequadamente quando necessário. Lembramos que na maioria dos casos a disfunção é apenas diastólica ${ }^{26}$.

\section{CONSIDERAÇÕES FINAIS E PERSPECTIVAS}

A AR deve ser considerada um fator de risco independente para doença arterial coronariana.

\section{REFERÊNCIAS}

1. Marques Neto JF, Gonçalves ET, Langen LFOB, Cunha MFL, Radominski S, Oliveira SM, et al. Multicentric study of the prevalence of adult rheumatoid arthritis in Brazilian population samples. Rev Bras Reumatol. 1993;33:169-73.

2. Haque S, Mirjafari H. Bruce IN. Atherosclerosis in rheumatoid arthritis and systemic lupus erythematosus. Curr Opin Lipidol. 2008;19:338-43.

3. Turesson C, Jarenros A, Jacobsson L. Increased incidence of cardiovascular disease in patients with rheumatoid arthritis: results from a community based study. Ann Rheum Dis. 2004;63:952-5.

4. Rindfleisch JA, Muller D. Diagnosis and management of rheumatoid arthritis. Am Fam Physician. 2005;72:103747.

5. Hürlimann D, Enseleit F, Ruschitzka F. Rheumatoide Arthritis, Inflammation und Atherosklerose. Herz 2004;29:760-8.

6. Gabriel SE. Heart disease and rheumatoid arthritis: understanding the risks. Ann Rheum Dis. 2010;69:i61i64.

7. Gabriel SE. Cardiovascular morbidity and mortality in rheumatoid arthritis. Am J Med. 2008;121:S9-14.

8. Gabriel SE, Crowson CS, Kremers HM, Doran MF, Turesson C, O'Fallon M, et al. Survival in rheumatoid arthritis: a population-based analysis of trends over 40 years. Arthritis Rheum. 2003;48:54-8.

9. Chung CP, Oeser A, Avalos I, Gebretsadik T, Shintani $A$, Raggi $P$, Sokka $T$, et al. Utility of the Framingham risk score to predict the presence of coronary atherosclerosis in patients with rheumatoid arthritis. Arthritis Res Ther. 2006;8:R186.

10. Sitia S, Atzeni F, Sarzi-Puttini P, Di Bello V, Tomasoni L, Delfino L, et al. Cardiovascular involvement in systemic autoimmune diseases. Autoimmun Rev. 2009;8:281-6.
Nos últimos anos houve um grande avanço no conhecimento relacionado à doenças auto-imunes $\mathrm{e}$ risco cardiovascular. No entanto, grande parte do conhecimento relacionado à doença cardiovascular em pacientes com AR ainda vem de estudos observacionais, sendo necessários novos trabalhos na área. Estratégias específicas de estratificação de risco e tratamento devem ser adotadas nesses pacientes. Portanto, novas recomendações devem surgir frente as atuais evidências. De maneira geral, o controle adequado da inflamação sistêmica é o principal fator envolvido na redução de risco de eventos coronarianos, devendo sempre ser alcançado.

11. Brenol CV, Monticielo OA, Xavier RM, Brenol JCT. Atrite reumatóide e aterosclerose. Rev Assoc Med Bras. 2007;53:465-70.

12. Gonzalez-Gay MA, Gonzalez-Juanatey C, Martin J. Rheumatoid arthritis: a disease associated with accelerated atherogenesis. Semin Arthristis Rheum. 2005;35:8-17.

13. Wolfe $F$, Michaud K, Heart failure in rheumatoid arthritis: rates, predictors, and the effect of anti-tumor necrosis factor therapy. Am J Med. 2004;116:305-311.

14. Gusmán AL, Díaz JDG, dela Vacas MID, Quintana EC, Atrio AS, Monzó IS. Coronary calcification and subclinical myocardial dysfunction in rheumatoid arthritis. Rev Clin Esp. 2009;209:428-32.

15. Maradit-Kremers $\mathrm{H}$, Crowson CS, Nicola PJ, Ballman KV, Roger VL, Jacobsen SJ, et al. Increased unrecognized coronary heart disease and sudden deaths in rheumatoid arthritis: a population-based cohort study. Arthritis Rheum. 2005;52:402-11.

16. Chung CP, Avalos I, Raggi P, Stein CM. Atherosclerosis and inflammation: insights from rheumatoid arthritis. Clin Rheumatol. 2007;26:1228-33.

17. Myasoedova E, Crowson CS, Kremers HM, et al. Total cholesterol and LDL levels decrease before rheumatoid arthritis. Ann Rheum Dis. 2010;69:1310-4.

18. Maradit-Kremers H, Nicola PJ, Crowson CS, Ballman KV, Jacobsen SJ, Roger VL, et al. Raised erythrocyte sedimentation rate signals heart failure in patients with rheumatoid arthritis. Ann Rheum Dis. 2007;66:76-80.

19. Nicola PJ, Maradit-Kremers H, Roger VL, Jacobsen SJ, Crowson CS, Ballman KV, et al. The risk of congestive heart failure in rheumatoid arthristis. Arthritis Rheum. 2005;52:412-20.

20. Liang KP, Kremers HM, Crowson CS, Snyder MR, Therneau TM, Roger VL, et al. Autoantibodies and the risk of cardiovascular events. J Rheumatol. 2009;36:2462-9. 
21. Giles JT, Szklo M, Post W, Petri M, Blumenthal RS, Lam G, et al. Coronary arterial calcification in rheumatoid arthritis: comparison with the Multi-Ethnic Study of Atherosclerosis. Arthritis Res Ther. 2009;11:R36.

22. Sodergen A, Stegmayr B, Lundberg V, Ohman ML, Jonsson SW. Increased incidence on and impaired prognosis after acute myocardial infarction among patients with seropositive rheumatoid arthristis. Ann Rheum Dis. 2007;66:263-6.

23. Aubry MC, Maradit-Kremers H, Reinalda MS, Crowson CS, Edwards WD, Gabriel SE. Differences in atherosclerotic coronary heart disease between subjects with and without rheumatoid arthritis. J Rheumatol. 2007;34:937-42.

24. Wang S, Yiu KH, Mok MY, Ooi GC, Khong PL, Mak KFH, et al. Prevalence and extent of calcification over aorta, coronary and carotid arteries in patients with rheumatoid arthritis. J Intern Med. 2009;266:445-52.

25. Crowson CS, Nicola PJ, Kremers HM, O'Fallon MO, Therneau TM, Jacobsen SJ, et al. How much of the increased incidence of heart failure in rheumatoid arthritis is atributable to traditional cardiovascular risk factors and ischemic heart disease? Arthritis Rheum. 2005;52:3039-44.

26. Liang KP, Myasoedova E, Crowson CS, Davis III JM, Roger VL, Karon BL, et al. Increased prevalence of diastolic dysfunction in rheumatoid arthritis. Ann Rheum Dis. 2010;69:1665-70.

27. Kao AH, Wasko MCM, Krishnaswami S, Wagner J, Edmundowicz D, Shaw P, et al. C-Reactive Protein and Coronary Artery Calcium in Asymptomatic Women With Systemic Lupus Erythematosus or Rheumatoid Arthritis. Am J Cardiol. 2008;102:755-60.

28. Davis JM, Kremers HM, Crowson CS, Nicola PJ, Ballman KV, Therneau TM, et al. Glucocorticoids and cardiovascular events in rheumatoid arthritis. Arthritis Rheum. 2007;56:820-30.

29. Park YB, Choi HK, Kim MY, Lee WK, Song J, Kim DK, et al. Effects of antirheumatic therapy on serum lipid levels in patients with rheumatoid arthritis: a prospective study. Am J Med. 2002;113:188-93.

30. Landewé RB, van den Borne BEEM, Breedveld FC, Dijkmans BAC. Methotrexate effects in patients with rheumatoid arthritis with cardiovascular comorbidity. Lancet. 2000;355:1616-7.

31. van Halm VP, Nurmohamed MT, Twisk JWR, Dijkmans BAC, Voskuyl AE. Disease-modifying antirheumatic drugs are associated with a reduced risk for cardiovascular disease in patients with rheumatoid arthritis: a case control study. Arthritis Res Ther. 2006;8:R151.

32. Choi HK, Hernán MA, Seeger JD, Robins JM, Wolfe F. Methotrexate and mortality in patients with rheumatoid arthritis: a porspective study. Lancet. 2002;359:11737.

33. Suissa S, Bernatsky S, Hudson M. Antiheumatic drug use and the risk of acute myocardial infarction. Arthritis Rheum. 2006;55:531-6.

34. Bosello S, Santoliquido A, Zoli A, Di Campli C, Flore R, Tondi $P$, et al. TNF-alpha blockade induces a reversible but transient effect on endothelial dysfunction in patients with long-standing severe rheumatoid arthritis. Clin Rheumatol. 2008;27:833-9.

35. Peters MJL, Vis M, van Halm VP, Wolbink GJ, Voskuyl $A E$, Lems WF, et al. Changes in lipid profile during infliximab and corticosteroid treatment in rheumatoid arthritis. Ann Rheum Dis. 2007;66:958-61.

36. Maki-Petaja KM, Booth AD, Hall FC, Wallace SML, Brown J, McEmery CM. Ezetimibe and simvastatin reduce inflammation, disease activity, and aortic stiffness and improve endothelial function in rheumatoid arthritis. J Am Coll Cardiol. 2007;50:852-8.

37. IV Diretriz Brasileira Sobre Dislipidemias e Prevenção da Aterosclerose. Departamento de Aterosclerose da Sociedade Brasileira de Cardiologia. Arq Bras Cardiol. 2007;88:1-19. 